\title{
A Novel Integrated Pharmacokinetic-Pharmacodynamic Model to Evaluate Combination Therapy and Determine In Vivo Synergism ${ }^{[\$}$
}

\author{
Young Hee Choi, Chao Zhang, Zhenzhen Liu, Mei-Juan Tu, Ai-Xi Yu, and Ai-Ming Yu \\ Department of Biochemistry and Molecular Medicine, University of California (UC) Davis School of Medicine, Sacramento, \\ California (Y.H.C., C.Z., Z.L., M.-J.T., A.-M.Y.); College of Pharmacy and Integrated Research Institute for Drug Development, \\ Dongguk University-Seoul, Ilsandong-gu, Goyang-si, Gyonggi-do, Republic of Korea (Y.H.C.); and Department of Orthopedic \\ Trauma and Microsurgery, Zhongnan Hospital of Wuhan University, Wuhan, Hubei, China (A.-X.Y.)
}

Received February 25, 2021; accepted March 9, 2021

\begin{abstract}
Understanding pharmacokinetic (PK)-pharmacodynamic (PD) relationships is essential in translational research. Existing PKPD models for combination therapy lack consideration of quantitative contributions from individual drugs, whereas interaction factor is always assigned arbitrarily to one drug and overstretched for the determination of in vivo pharmacologic synergism. Herein, we report a novel generic PK-PD model for combination therapy by considering apparent contributions from individual drugs coadministered. Doxorubicin (Dox) and sorafenib (Sor) were used as model drugs whose PK data were obtained in mice and fit to two-compartment model. Xenograft tumor growth was biphasic in mice, and PD responses were described by three-compartment transit models. This PK-PD model revealed that Sor (contribution factor $=1.62$ ) had much greater influence on overall tumor-growth inhibition than coadministered Dox (contribution factor $=0.644$ ), which explains the mysterious clinical findings on remarkable benefits for patients with cancer when adding Sor to Dox treatment, whereas there were none when adding Dox to Sor therapy. Furthermore, the combination index method was integrated into this predictive PK-PD model for critical determination of in vivo pharmacologic synergism that cannot be correctly defined
\end{abstract}

by the interaction factor in conventional models. In addition, this new PK-PD model was able to identify optimal dosage combination (e.g., doubling experimental Sor dose and reducing Dox dose by $50 \%$ ) toward much greater degree of tumor-growth inhibition (>90\%), which was consistent with stronger synergy (combination index $=0.298$ ). These findings demonstrated the utilities of this new PK-PD model and reiterated the use of valid method for the assessment of in vivo synergism.

\section{SIGNIFICANCE STATEMENT}

A novel pharmacokinetic (PK)-pharmacodynamic (PD) model was developed for the assessment of combination treatment by considering contributions from individual drugs, and combination index method was incorporated to critically define in vivo synergism. A greater contribution from sorafenib to tumorgrowth inhibition than that of coadministered doxorubicin was identified, offering explanation for previously inexplicable clinical observations. This PK-PD model and strategy shall have broad applications to translational research on identifying optimal dosage combinations with stronger synergy toward improved therapeutic outcomes.
A.-M.Y. is supported by National Institutes of Health National Cancer Institute [Grant R01-CA225958]. Y.H.C. is funded by the National Research Foundation of Korea [Grants NRF-2016R1C1B2010849 and NRF2018R1A5A2023127l, and A.-X.Y. is supported by Hubei Province Scientific and Technological Innovation Key Project (2019ACA136) and Hubei Province Medical Leader Talent Project (LJ20200405). The authors also appreciate the access to the Molecular Pharmacology Shared Resources funded by the University of California (UC) Davis Comprehensive Cancer Center Support Grant awarded by National Institutes of Health National Cancer Institute [Grant P30-CA093373].

No author has an actual or perceived conflict of interest with the contents of this article.

https://doi.org/10.1124/jpet.121.000584.

S This article has supplemental material available at jpet.aspetjournals.org.

\section{Introduction}

Integrated pharmacokinetic (PK)-pharmacodynamic (PD) model provides quantitative information for understanding drug exposure-response relationship. Besides a variety of dose- or concentration-response relationships, complex disease dynamics or effects over time in response to a particular dosage of drug may be described by proper PK-PD models that may not only help to understand important factors or mechanisms underlying drug actions but also predict possible drug effects vis-a-vis different dose regimens toward dosage optimization (Mager et al., 2003; Altrock et al., 2015; Mould et al., 2015; Li et al., 2019; Ayyar and Jusko, 2020). PK-PD modeling has been applied to essentially all phases of drug development,

ABBREVIATIONS: AIC, Akaike information criterion; AUC, area under the curve; Cl, combination index; Combo, combination; Dox, doxorubicin; IS, internal standard; $I V$ intraveneous $L_{0}$, coefficient of the exponential growth phase; $L_{1}$, slope of the linear growth phase; LC-MS/MS, liquid chromatography tandem mass spectrometry; MSE, mean square error; $\mathrm{m} / \mathrm{z}$ mass to charge ratio PD, pharmacodynamics; $\mathrm{PK}$, pharmacokinetics; p.o., by mouth; Sor, sorafenib; SSE, sum of squared error; TEl, time efficacy index. 
types of diseases, and forms of drugs for improved therapy or precision medicine. In addition, recommendations have been documented by the United States Food and Drug Administration (https://www.fda.gov/media/71277/download) to guide sponsors to identify and use exposure-response information in the development of new therapeutics.

Many PK-PD models have been developed in oncology to describe the dynamics of tumor growth or biomarkers in animal models as well as patients with cancer subjected to various types of medications consisting of empirical, indirect response or particular hypothesis- or mechanism-based PD models (Laird, 1964; Simeoni et al., 2004; Li et al., 2008; Tanaka et al., 2008; Claret et al., 2009; Wada et al., 2014; Schindler et al., 2016; Diekstra et al., 2017; Cho et al., 2018; Iida et al., 2020; Vaghi et al., 2020). Rather, it is a common practice to use multiple medications for the treatment of patients with cancer, and many other new combinations are under active development (Webster, 2016). Since experimental approach has many limitations to test numerous possible combinations of various dosage regimens, PK-PD modeling holds great promise in assessing combination therapy and determining optimal combination. Indeed, there are a number of integrated PK-PD models being developed to characterize antitumor efficacy of coadministered drugs (Koch et al., 2009; Rocchetti et al., 2009; Pawaskar et al., 2013; Terranova et al., 2013; Yuan et al., 2015; Li et al., 2016; Nanavati and Mager, 2017; Chen et al., 2018). Among them, an interaction term represented by $\psi$ is commonly used to indicate the nature and intensity of drug interactions and determine the combined outcome (Koch et al., 2009). It is obvious that the interaction factor $\psi$ may be assigned to either drug $\mathrm{A}$ or $\mathrm{B}$, which undoubtedly leads to two possibilities (Fig. 1). However, the interaction factor was only allocated to a particular drug in all previous studies (Koch et al., 2009; Pawaskar et al., 2013; Yuan et al., 2015; Li et al., 2016; Nanavati and Mager, 2017; Chen et al., 2018), either using the precision error value as a criterion or without providing any pharmacologic justifications. Concerns remain because the precision error values in two cases are close to each other.

In addition, although the $\psi$ value is able to indicate the degree of change in antitumor effect for combination treatment (Koch et al., 2009), it has been overstretched for the determination of in vivo pharmacologic synergism (i.e., when $\psi>1$ ) in previous studies (Koch et al., 2009; Pawaskar et al., 2013; Yuan et al., 2015; Li et al., 2016; Nanavati and Mager, 2017; Chen et al., 2018). The confusion of pharmacologic synergy with enhancement or potentiation of efficacy ignores the concept of dose equivalence in assessing combination therapy, which emphasizes the utilization of valid approaches and algorisms for accurate determination of synergistic, additive, or antagonistic effects (Chou, 2006; Tallarida, 2006; Chou, 2010). Unlike the determination of in vitro synergism in cells (Jilek et al., 2020; Yi et al., 2020), which is relatively more straightforward and less expensive, experimental determination of in vivo synergy requires not only proper study design but also application of a large number of animals (Fu et al.,

\section{B Model B: PK-PD of Sor (p.o.) monotherapy}

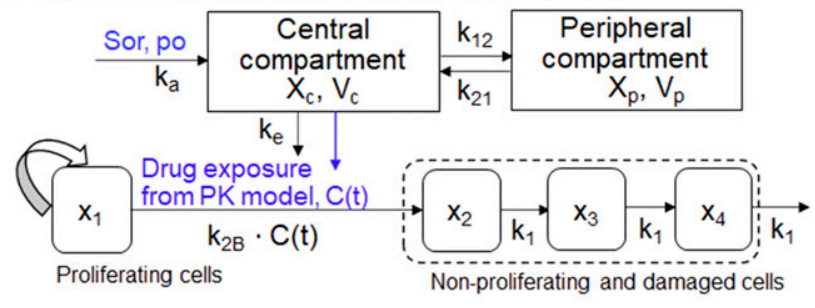

D Model D: New PK-PD model for combination therapy (established in this study)

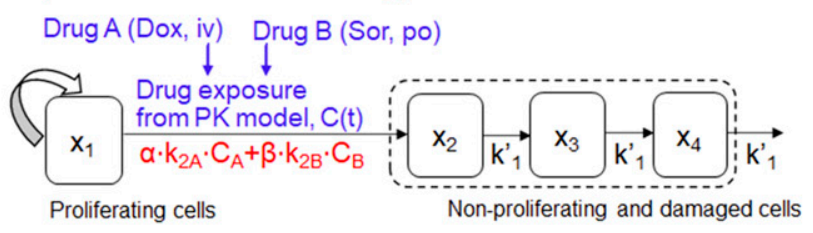

Combination index $(\mathrm{Cl})$ is precisely determined by using Chou-Talalay method to critically define the combination effects.

- $\mathrm{Cl}>1$, antagonism

- $\mathrm{Cl}=1$, addition

- $\mathrm{Cl}<1$, synergism

Fig. 1. Integrated PK-PD models of Dox [intravenous; (A)] and Sor [p.o.; (B)] monotherapy as well as combination therapy with both drugs (C and D) in mouse models. Not that conventional PK-PD model of combination therapy actually poses two possibilities: Models C1 and C2, in which the interaction factor $\psi$ may be assigned to drug A or B. As such, models C1 and C2 will undoubtedly lead to different results and predictions (see the Results). Herein we introduce a new model $\mathrm{D}$ by considering contributions from individual drugs $\left(\alpha \cdot \mathrm{k}_{2 \mathrm{~A}} \cdot \mathrm{C}_{\mathrm{A}}\right.$ and $\beta \cdot \mathrm{k}_{2 \mathrm{~B}} \cdot \mathrm{C}_{\mathrm{B}}$, etc.) that can be represented by the contribution factors $\alpha$ and $\beta$, etc. Our new model offers one definitive result for the same dose combination. In addition, whether drug combination produces a synergistic, additive, or antagonistic effect should be critically determined by using a proper approach (e.g., Chou-Talalay method or the identical isobologram approach) because the $\psi$ value just signifies the degree of change in responses. See Materials and Methods for specific differential equations and definitions of individual parameters. 
2016), which is time-consuming and costly. Combining predictive PK-PD model and proper method for quantitative determination of pharmacologic synergism shall be useful for the investigation of combination therapy in vivo.

In this study, we developed a new generic PK-PD model (Fig. 1D) for the evaluation of combination treatment by considering apparent contributions of individual drugs coadministered, which are denoted by the contribution factors $(\alpha$ and $\beta$, etc.) and may be interpreted as the degrees of changes in efficacy altered by coadministered drug(s). The combination of doxorubicin (Dox; drug A) and sorafenib (Sor; drug B) was used to evaluate this new PK-PD model in comparison with conventional model (Fig. 1C). Our new PK-PD model disclosed a greater contribution from Sor to the antitumor efficacy than Dox during combination therapy, which directly explains the mysterious findings on the remarkable improvement of clinical outcomes when adding Sor to Dox treatment, whereas there was an absence of benefits when adding Dox to Sor treatment in patients with advanced hepatocellular carcinoma (Abou-Alfa et al., 2010, 2019). Simulation studies were further conducted to investigate optimal dosage combination. In addition, we incorporated the Chou-Talalay method (Chou, $2006,2010)$ into our predictive PK-PD model for the calculation of combination index (CI) to critically determine in vivo synergism, additivity, and antagonism and exemplified the misuse of interaction factor $\psi$ in conventional PK-PD model for the evaluation of pharmacologic synergy.

\section{Materials and Methods}

Chemicals and Reagents. Dox (hydrochloride salt; $>99 \%$ ), Sor [ $p$-toluenesulfonate salt (the other name tosylate salt used in the clinic); $>99 \%$ ], and daunorubicin (hydrochloride salt; $>98 \%$ ) were purchased from LC Laboratories (Woburn, MA). All drugs were verified by liquid chromatography tandem mass spectrometry (LC-MS/MS) analyses, and the same lots of drugs were used for bioanalytical method development and pharmacokinetic studies herein as well as previous therapeutic studies (converted and unified as the amounts of free bases in mass or mole units) (Jian et al., 2017). All other chemicals and organic solvents of analytical grade were purchased from Sigma-Aldrich (St. Louis, MO) or Thermo-Fisher Scientific Inc. (Waltham, MA).

Animals. All animal procedures were approved by the Institutional Animal Case and Use Committee of University of California at Davis (protocols 21155 and 19396), and only trained and experienced individuals approved by the Institutional Animal Case and Use Committee conducted animal procedures. All animal studies were conducted in accordance with the Guide for the Care and Use of Laboratory Animals recommended by the National Research Council (US) Committee for the Update of the Guide for the Care and Use of Laboratory Animals (2011). Male athymic nude mice (Athymic NudeFoxn $1^{\text {nu }} ; 7$ weeks old, approximately $30 \mathrm{~g}$ body weight) were purchased from Envigo (Hayward, CA). All animals were group-housed in individually ventilated cages (2-4 per cage) (Tecniplast, West Chester, PA) at an institutional animal facility certified by The Association for Assessment and Accreditation of Laboratory Animal Care International under 12-hour controlled light/dark conditions, temperature $\left(72^{\circ} \mathrm{F}\right)$, humidity $(20 \%-40 \%)$, and room air circulation and supplied $\mathrm{ad}$ libitum with regular diet (Teklad 2918; Envigo) as well as sterile and distilled water via Hydropacs. All cages, bedding (corn cob), enrichment (Enviro-dri), feeder, and filter top were autoclaved before use. After arrival, all animals were adaptively housed in the facility at least 1 week before the experiments. After the PK experiments were completed, all mice (18 in total) were euthanized by overdose inhalation of carbon dioxide.
PK Studies. To characterize the PK properties of Dox, $0.06 \mathrm{mg}$ of Dox (dissolved in distilled water as doxorubicin hydrochloride) was administered intravenously into each mouse $(n=6)$ via tail-vein injection. A $15-\mu 1$ blood sample was collected at each time point ( $0,0.083,0.167,0.5,1,3,5,8,12$, and 24 hours) from individual mice after Dox injection through microsampling, as we described recently (Jilek et al., 2017). Likewise, $0.02 \mathrm{mg}$ of Sor (dissolved in polyoxyethylated castor oil as sorafenib $p$-toluensulfonate) was administered intravenously into another group of mice $(n=6)$ via tail-vein injection. A $15-\mu \mathrm{l}$ blood sample was collected at various time points $(0,0.083$, $0.167,0.5,1,3,5,8,11,24$, and 48 hours) after Sor administration. A third group of mice $(n=6)$ was treated with $0.2 \mathrm{mg}$ of Sor via oral gavage (p.o.), and blood samples were collected at 0, 0.5, 1, 2, 3, 4, 6, 11 , 24 48, 72, and 96 hours after Sor administration. All blood samples were transferred into heparinized microcentrifuge tubes and immediately centrifuged at $3300 \mathrm{~g}$ for 5 minutes, and plasma samples were isolated and stored at $-80^{\circ} \mathrm{C}$ until further analyses.

LC-MS/MS Analyses. Quantification of plasma drug concentrations was conducted on a Shimadzu Prominence Ultra-Fast Liquid Chromatography system (Shimadzu Corporation, Kyoto, Japan) coupled with an AB Sciex 4000 QTRAP mass spectrometer consisting of an electrospray ionization source (AB SCIEX, Framingham, MA). For the analysis of Dox, $8 \mu \mathrm{l}$ of plasma was deproteinized with $50 \mu \mathrm{l}$ of acetonitrile containing $10 \mathrm{ng} / \mathrm{ml}$ of daunorubicin [internal standard (IS)]. After vortex mixing and centrifugation, the supernatant was transferred into a new vial from which $5 \mu \mathrm{l}$ was directly injected for LC-MS/MS analysis. The mobile phase consisted of water with $0.1 \%$ formic acid (solution A) and acetonitrile with $0.1 \%$ formic acid (solution B) running at a constant rate of $0.45 \mathrm{ml} / \mathrm{min}$. An optimal gradient elution was developed for the separation of Dox on a reversephase $\mathrm{C}_{18}$ column (Phenomenex Luna, $2.0 \times 100 \mathrm{~mm}$ i.d., $3.0 \mu \mathrm{m}$ particle size, Phenomenix) maintained at $45^{\circ} \mathrm{C}$ : 0-1.0 minutes, $10 \%-$ $24 \%$ solution $\mathrm{B} ; 1.0-3.0$ minutes, $24 \%-28 \%$ solution $\mathrm{B} ; 3.0-5.5$ minutes, $28 \%-90 \%$ solution $\mathrm{B}$; and $5.5-5.6$ minutes, $90 \%-10 \%$ solution $\mathrm{B}$, with a total run time of 8 minutes. The ion source was operated in positive mode under an optimal condition: curtain gas, 25 psi; nebulizer gas, $40 \mathrm{psi}$; auxiliary gas, $45 \mathrm{psi}$; ion spray voltage, 1500 $\mathrm{V}$; and temperature, $600^{\circ} \mathrm{C}$. Optimal multiple reaction monitoring transition was mass to charge ratio $(\mathrm{m} / z)[\mathrm{M}+\mathrm{H}]^{+} 544.2 \rightarrow 397.2(51$ and $19 \mathrm{~V}$, respectively) for Dox and $m / z[\mathrm{M}+\mathrm{H}]^{+} 528.1 \rightarrow 321.1$ (51 and $29 \mathrm{~V}$, respectively) for the IS. The retention times of Dox and IS were 3.35 and 4.90 minutes, respectively.

For the analysis of Sor, $8 \mu \mathrm{l}$ of plasma was deproteinized with $64 \mu \mathrm{l}$ of acetonitrile containing $80 \mathrm{ng} / \mathrm{ml}$ of XY063 as the IS (Wang et al., 2016). After vortex mixing and centrifugation, the supernatant was transferred into a new vial, and then $5 \mu \mathrm{l}$ was directly injected for LC-MS/MS analysis. The mobile phase consisted of water containing $5 \mathrm{mM}$ ammonium formate and $0.1 \%$ formic acid (solution $\mathrm{C}$ ) and methanol with $5 \mathrm{mM}$ ammonium formate and $0.1 \%$ formic acid (solution D) at a flow rate of $0.6 \mathrm{ml} / \mathrm{min}$. A gradient elution was optimized and employed for the separation of Sor on a reverse-phase $\mathrm{C}_{18}$ column (Phenomenex F5, $2.1 \times 50 \mathrm{~mm}$ i.d., $2.6 \mu \mathrm{m}$ particle size, Phenomenix) maintained at $40^{\circ} \mathrm{C}: 0-4.0$ minutes, $0 \%-90 \%$ solution $\mathrm{D}$; 4.0-6.0 minutes, $90 \%-100 \%$ solution $\mathrm{D}$; $6.0-7.0$ minutes, $100 \%-$ $100 \%$ solution D; and 7.0-10.0 minutes, $100 \%-0 \%$ solution $\mathrm{D}$, with a total run time of 10 minutes. The ion source was operated in positive mode under an optimal condition: curtain gas, 25 psi; nebulizer gas, 40 psi; auxiliary gas, $45 \mathrm{psi}$; ion spray voltage, $1500 \mathrm{~V}$; and temperature, $600^{\circ} \mathrm{C}$. Optimal multiple reaction monitoring transition was $\mathrm{m} / z[\mathrm{M}+$ $\mathrm{H}]^{+} 465.0 \rightarrow 251.9$ (90 and $45 \mathrm{~V}$, respectively) for Sor and $m / z[\mathrm{M}+\mathrm{H}]^{+}$ $478.1 \rightarrow 187.1$ (110 and $70 \mathrm{~V}$, respectively) for the IS. The retention times of Sor and IS were 3.04 and 3.12 minutes, respectively.

Dox and Sor Monotherapy and Combination Therapy in Xenograft Mouse Models. Antitumor effects of Dox (intravenous), Sor (p.o.), and their combination (Combo) treatments determined in female OD.CB17-Prkdcscid/J mice bearing orthotopic human osteosarcoma 143B cell line-derived xenografts ( $n=7$ /group) were reported separately (Jian et al., 2017). In this study, all raw data of tumor 
volumes in mice subjected to Dox, Sor, and control treatments $(n=7 /$ group) were used to establish natural tumor-growth characteristics and the PK-PD relationship of Dox and Sor monotherapy (Fig. 1, A and B). The tumor-growth data of five xenograft mice $(n=5)$ within the Combo treatment group were randomly chosen and used for the development of new PK-PD model of combination therapy and comparison of modeling as well as prediction results with conventional models (Fig. 1, C and D), whereas the rest of the Combo data $(n=2)$ were employed for initial verification of this new model (Fig. 1D).

Integrated PK-PD Modeling and Simulation. A twocompartment model with a linear elimination (Fig. 1A) was fit to Dox PK data (Fig. 2A). Differential equations for the two-compartment model of Dox (intravenous) PK were as follows:

$$
\begin{gathered}
\frac{d X_{c}}{d t}=-k_{12} \cdot X_{c}-k_{e} \cdot X_{c}+k_{21} \cdot X_{p} \\
\frac{d X_{p}}{d t}=k_{12} \cdot X_{c}-k_{21} \cdot X_{p}
\end{gathered}
$$

in which $k_{12}$ ( 1 per hour) and $k_{21}$ ( 1 per hour) are the apparent firstorder intercompartmental distribution constants (or transfer rate constants), $k_{\mathrm{e}}$ (1 per hour) is the apparent first-order elimination rate constant from the central compartment, and $X_{\mathrm{c}}$ (milligram) and $X_{\mathrm{p}}$ (milligram) are the amounts of drug in central and peripheral compartments, respectively.

Likewise, a two-compartment model with first-order absorption and a linear elimination (the upper part in Fig. 1B) was revealed to better describe Sor (p.o.) PK profiles (Fig. 2A). This model was sequentially fit to the intravenous and p.o. data to offer specific PK parameters: $V_{\mathrm{c}}$,
$V_{\mathrm{p}}, k_{12}, k_{21}, k_{\mathrm{e}}$, and $k_{\mathrm{a}}$. The differential equations of the final model were as follows:

$$
\begin{gathered}
\frac{d A}{d t}=-k_{a} \cdot A \\
\frac{d X_{c}}{d t}=-k_{a} \cdot A-k_{12} \cdot X_{c}-k_{e} \cdot X_{c}+k_{21} \cdot X_{p} \\
\frac{d X_{p}}{d t}=k_{12} \cdot X_{c}-k_{21} \cdot X_{p}
\end{gathered}
$$

in which $k_{\mathrm{a}}$ ( 1 per hour) is the apparent first-order absorption rate constant to the central compartment, and $A$ (milligram) is the drug amount in absorption site. All other parameters for Sor share the same definition as Dox described above.

The established PK model was thus coupled with corresponding PD models comprised of an empirical tumor natural-growth model (Simeoni et al., 2004) (Fig. 1, A and B). The empirical tumor natural-growth model assumes the presence of two phases of tumor growth: an initial exponential growth followed by a subsequent linear growth, which is indeed obvious in our studies (Fig. 2A). Tumor growth switches from exponential to linear phase of growth at the threshold tumor volume (or mass, $\mathrm{w}_{\text {th }}$ ) (Simeoni et al., 2004; Koch et al., 2009). Differential equations for tumor natural-growth model are shown as follows:

$$
\begin{gathered}
\frac{d x_{1}}{d t}=\frac{2 \cdot L_{0} \cdot L_{1} \cdot x_{1}}{L_{1}+2 \cdot L_{0} \cdot x_{1}} \\
x_{1}(0)=w_{0}
\end{gathered}
$$

A

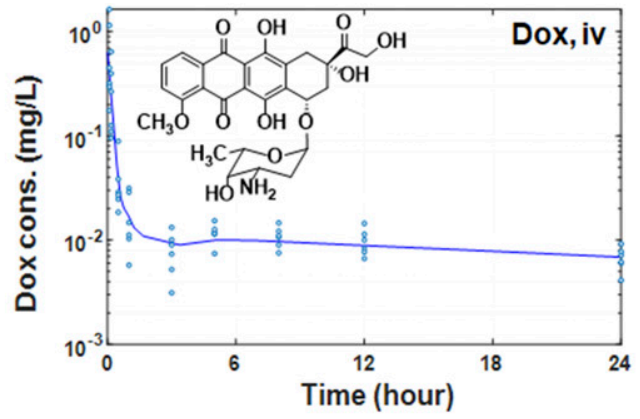

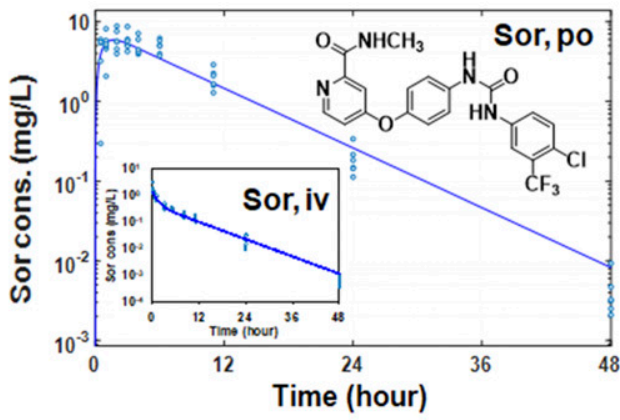
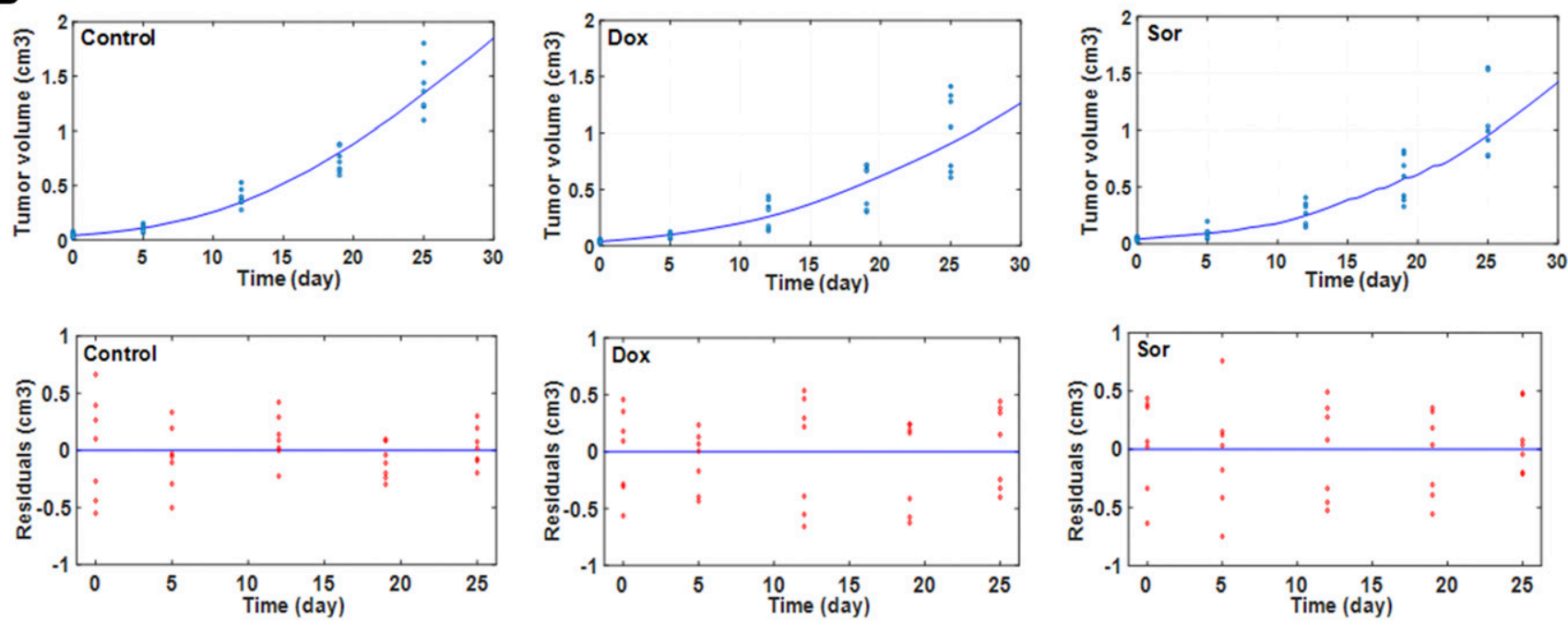

Fig. 2. Experimental and model-predicted PK and PD profiles in mice subjected to Dox or Sor monotherapy. (A) Plasma drug concentrations vs. time curves in mice treated with a single dose of Dox (iv) or Sor (p.o.) $(n=6$ /group). The insert shows plasma Sor concentrations after intravenous administration $(n=6)$. (B) Observed and predicted tumor-growth profiles and corresponding residual time plots for mice treated with multiple doses of Dox, Sor, or vehicle control ( $n=7$ /group). See Supplemental Fig. 1 for the simulated plasma drug concentration vs. time curves in mice treated with multiple doses. 
A
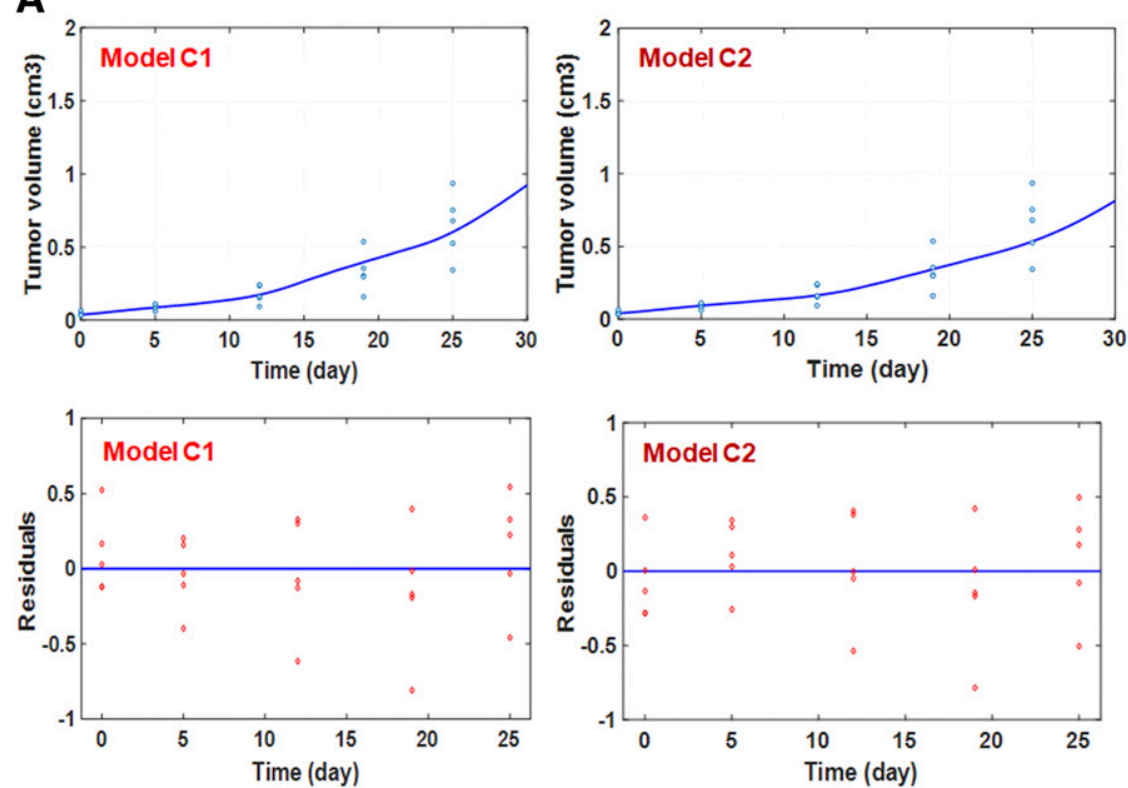

B
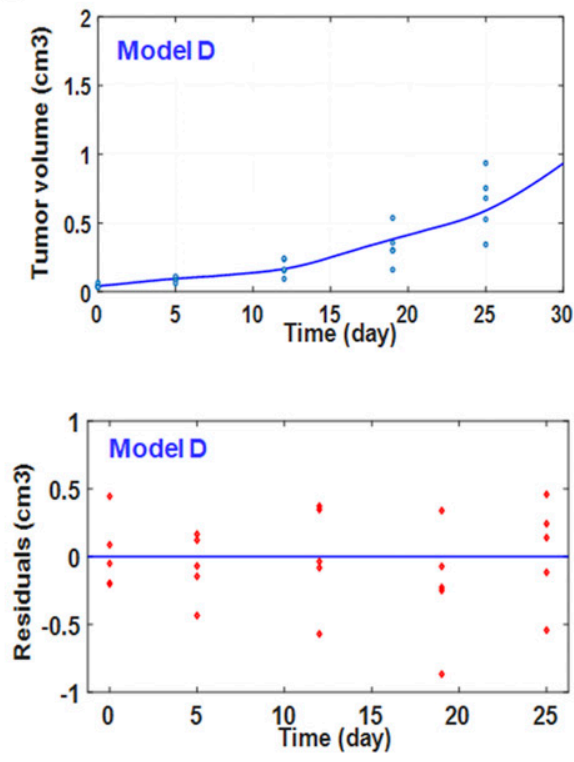

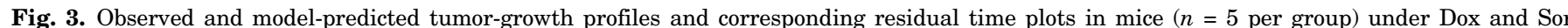

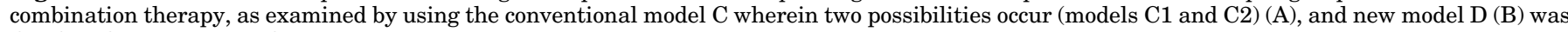
developed in current study.

in which $L_{0}$ ( 1 per day) is the first-order growth rate constant of the exponential growth phase, $L_{1}$ (cubic centimeter per day) denotes the zero-order growth rate of the linear growth phase, $x_{1}$ (cubic centimeter) represents the proliferating tumor volume, and $w_{0}$ (cubic centimeter) represents the initial tumor volume.

The PK-PD model for Dox and Sor monotherapy was established by considering that drug treatment destroys some tumor cells and changes them to nonproliferating cells $\left(x_{2}, x_{3}, x_{4}\right.$, etc. $)$ that are eventually killed after a certain number of damage states, whereas vehicle treatment (as the control) does not cause any changes of natural growth, and all cells are proliferating $\left(x_{1}\right)$ (Fig. 1, A and B). This PK-PD model is able to capture anticancer effect of drug in which the suppression of tumor growth usually shows a delay. Two parameters, $k_{1}$ (the transient rate constant linked to the death delay) and $k_{2}$ (the drug potency), describe the relationship between the drug concentrations and anticancer effects. The average time to death of damaged cells is represented by $N / k_{1}(N$; a number of transit compartment describing the number of stages of damaged cells) (Simeoni et al., 2004). Three-compartment transits (i.e., $N=3$; Fig. 1, A and B) were found to nicely capture experimental data (Fig. 2B) and thus were used in this study. Differential equations for PK-PD model of Dox or Sor monotherapy are shown as follows:

$$
\begin{gathered}
\frac{d x_{1}}{d t}=\frac{2 \cdot L_{0} \cdot L_{1} \cdot x_{1}^{2}}{\left(L_{1}+2 \cdot L_{0} \cdot x_{1}\right) \cdot w}-k_{2} \cdot C \cdot x_{1} \\
\frac{d x_{2}}{d t}=k_{2} \cdot C \cdot x_{1}-k_{1} \cdot x_{2} \\
\frac{d x_{3}}{d t}=k_{1} \cdot x_{2}-k_{1} \cdot x_{3} \\
\frac{d x_{4}}{d t}=k_{1} \cdot x_{3}-k_{1} \cdot x_{4} \\
w=x_{1}+x_{2}+x_{3}+x_{4} \\
x_{2}(0)=x_{3}(0)=x_{4}(0)=0 \\
x_{1}(0)=w_{0}
\end{gathered}
$$

in which $x_{1}$ (cubic centimeter) is the proliferating tumor volume; $x_{2}, x_{3}$, and $x_{4}$ (cubic centimeter) are damaged or quiescent tumor volumes; $k_{1}$
( 1 per day) is the transient rate constant; $k_{2}$ (liter per milligram per day) is the potency of the drug; and $C$ (milligram per liter) is drug concentration.

The control treatment data were fit to the tumor natural-growth model to determine the $L_{0}$ and $L_{1}$ values, and Dox and Sor monotherapy data were subsequently modeled to offer $w_{0}, k_{1}$, and $k_{2}$ values for individual drugs. The simulated Dox or Sor concentrations in mice administered with multiple doses of drug (Supplemental Fig. 1, A and B) were used to drive the inhibition of tumor growth (Fig. 2B).

To model the combination therapy, we first noticed that there are two options when using the interaction term introduced by Koch et al. (2009) and used by many other investigators (Pawaskar et al., 2013; Yuan et al., 2015; Li et al., 2016; Nanavati and Mager, 2017; Chen et al., 2018) (Fig. 1C). The interaction factor $\psi$ may be applied to either drug A (Dox) or drug B (Sor), which seems arbitrary and undoubtedly leads to different results (models $\mathrm{C} 1$ and $\mathrm{C} 2$ in Fig. 3A and Table 3; and Results). Corresponding differential equations are shown in Supplemental Methods.

The new PK-PD model (model D; Fig. 1D) reported in this study takes into consideration the actual weighed contributions ( $\alpha$ and $\beta$ ) from individual drugs (drug A and B) during combination treatment, which might be affected by each other, whereas potency of each drug $\left(k_{2 \mathrm{~A}}\right.$ and $\left.k_{2 \mathrm{~B}}\right)$ remains constant.

$$
\begin{gathered}
\frac{d x_{1}}{d t}=\frac{2 \cdot L_{0} \cdot L_{1} \cdot x_{1}^{2}}{\left(L_{1}+2 \cdot L_{0} \cdot x_{1}\right) \cdot w}-\left[\alpha \cdot k_{2 A} \cdot C_{A}+\beta \cdot k_{2 B} \cdot C_{B}\right] \cdot x_{1} \\
\frac{d x_{2}}{d t}=\left[\alpha \cdot k_{2 A} \cdot C_{A}+\beta \cdot k_{2 B} \cdot C_{B}\right] \cdot x_{1}-k_{1}^{\prime} \cdot x_{2} \\
\frac{d x_{3}}{d t}=k_{1}^{\prime} \cdot x_{2}-k_{1}^{\prime} \cdot x_{3} \\
\frac{d x_{4}}{d t}=k_{1}^{\prime} \cdot x_{3}-k_{1}^{\prime} \cdot x_{4} \\
w=x_{1}+x_{2}+x_{3}+x_{4} \\
x_{2}(0)=x_{3}(0)=x_{4}(0)=0 \\
x_{1}(0)=w_{0}
\end{gathered}
$$

in which $k^{\prime}{ }_{1}$ is a transient rate constant in combination therapy, and $\alpha$ and $\beta$ are contribution factors of individual drugs (Dox and Sor 
TABLE 1

PK parameters estimated for Dox and Sor, with \%CV (shown in parentheses) ( $n=6$ /group)

Respective objective function values are included.

\begin{tabular}{lrrr}
\hline & Dox (iv) & \multicolumn{1}{c}{ Sor (iv) } & Sor (p.o.) $^{\mathrm{a}}$ \\
\hline Estimated parameters $^{\mathrm{a}}$ & & & \\
$V_{\mathrm{c}}[\mathrm{l}](\% \mathrm{CV})$ & $0.0764(23.4)$ & $0.0112(9.92)$ & 0.0112 (fixed) \\
$V_{\mathrm{p}}[\mathrm{l}](\% \mathrm{CV})$ & $3.52(27.1)$ & $0.0120(13.7)$ & 0.0120 (fixed) \\
$k_{12}[1 / \mathrm{h}](\% \mathrm{CV})$ & $5.86(19.1)$ & $0.663(4.06)$ & 0.663 (fixed) \\
$k_{21}[1 / \mathrm{h}](\% \mathrm{CV})$ & $0.127(19.1)$ & $0.620(9.19)$ & $0.620($ fixed) \\
$k_{\mathrm{e}}[1 / \mathrm{h}](\% \mathrm{CV})$ & $1.24(37.1)$ & $0.344(31.4)$ & $0.344($ fixed) \\
$k_{\mathrm{a}}[1 / \mathrm{h}](\% \mathrm{CV})$ & & & $0.640(18.8)$ \\
Objection function & & & \\
values & & & 0.337 \\
MSE & 0.290 & 0.0741 & 97.9 \\
SSE & 14.5 & 4.15 & 95.5 \\
AIC & 90.3 & 18.1 & \\
\hline
\end{tabular}

$k_{12}$ or $k_{21}$, apparent first-order intercompartmental distribution or transfer rate constants; $k_{\mathrm{a}}$, apparent first-order absorption rate constant to the central compartment; $k_{\mathrm{e}}$, apparent first-order elimination rate constant from the central compartment; $V_{\mathrm{c}}$ or $V_{\mathrm{p}}$, volume of distribution in central or peripheral compartment.

${ }^{a}$ Only the $k_{\mathrm{a}}$ value was estimated for Sor (p.o.), whereas others were fixed as the same as Sor (i.v.).

herein, respectively). Other parameters remain the same as monotherapy, as described above.

All modeling and simulation were conducted by using SimBiology in MATLAB 2019a (The MathWorks, Inc.). Predicted data were obtained after 400 times simulation. Model selection and evaluation was based on goodness-to-fit criteria, which included model convergence, mean square error (MSE), sum of squared error (SSE), Akaike information criterion (AIC), values visual inspection of predicted versus observed values, and residual plots as well as physiologic plausibility. Different error models (e.g., constant, proportional, combined, and exponential error models) were also tested during model development, and the exponential model showing better goodness of fit was used in final model. The source MATLAB files are available online.

This developed PK-PD model (Fig. 1D) was externally verified by comparing the simulated and experimentally determined tumor progression profiles in two mice randomly left from the total seven mice (Jian et al., 2017), as the other five were used for model development. For model verification, visual inspection of observed and predicted data, linear regression, and Pearson correlation were considered. Simulations were further conducted by using model C (including models $\mathrm{C} 1$ and $\mathrm{C} 2$ ) and model $\mathrm{D}$ to obtain the tumor-growth profiles in response to various combinations of Dox and Sor doses.

Assessment of Combination Effects. CI values were determined to critically define the combination effects (i.e., CI $<1$ indicates synergism, $\mathrm{CI}=1$ shows additivity, and $\mathrm{CI}>1$ points to antagonism) for respective dose combinations by using the Chou-Talalay method (Chou, 2010) and predictive PK-PD model (Fig. 1). Specifically, individual CI values relevant to the fraction affected $\left(\mathrm{F}_{\mathrm{a}}\right)$ for various dose combinations were calculated with CompuSyn software (ComboSyn, Inc.).

\section{Results}

PK-PD Models for Dox and Sor Monotherapy and the Estimations. After the intravenous and oral administration, plasma Dox and Sor concentrations versus time curves were both established in mice (Fig. 2A), which were readily described by two-compartment PK model (Fig. 1, A and B). Oneand three-compartment models were not fit well to the PK data simply through visual inspection and according to quantitative goodness-to-fit criteria. Considering that tumorgrowth inhibition showed a delay (Fig. 2B) as compared with the changing patterns of drug concentrations, transient compartments with the first-order transit rate (Simeoni et al., 2004) were used in PD modeling (Fig. 1). Three-compartment transit model was revealed to reasonably characterize tumorgrowth inhibition in our studies. The nal PK-PD models of Dox and Sor monotherapy (Fig. 1, A and B) were able to describe well all experimental PK data (Fig. 2A) and tumor progression profiles in mice subjected to Dox, Sor, and control treatments (Fig. 2B). The respective PK (Table 1) and PD parameters (Table 2) were estimated with good precisions, as manifested by the low CV values (\%) as well as other indicators, such as MSE, SSE, and AIC.

The tumor progression profiles in control mice and monotherapy groups (i.e., Dox and Sor) all followed a natural tumor-growth pattern with two distinct growth phases that were captured by the estimated $L_{0}(0.1071$ /day $)$ and $L_{1}$ $\left(0.148 \mathrm{~cm}^{3} /\right.$ day) values (Table 2$)$. The $k_{2 \mathrm{~A}}$ of Dox $(13.8 \mathrm{l} / \mathrm{mg}$ per day) was revealed to be much greater than $k_{2 \mathrm{~B}}$ of Sor $(0.0267 \mathrm{l} / \mathrm{mg}$ per day), indicating that Dox is more potent than Sor for the inhibition of xenograft tumor growth. By contrast, the transition rate constant $k_{1}$ value of Dox $(0.1301$ day $)$ was found to be much smaller than that of Sor (17.0 1/day), suggesting that it would take a longer time for nonproliferating tumor cells to become dead after Dox treatment than after Sor. Indeed, the average time to death of damaged cells calculated by using the formula $N / k^{\prime}{ }_{1}$ (Simeoni et al., 2004) was 23.1 and 0.176 days for Dox and Sor monotherapy, respectively, indicating that tumor cells are induced to death much more quickly by Sor than Dox.

Development of a New PK-PD Model for Combination Therapy and the Estimations. We first noted that the interaction factor $\psi$ within conventional PK-PD model of combination therapy (Fig. 1C) may be assigned to either drug (i.e., $\Psi_{\text {Dox }}$ and $\Psi_{\text {Sor }}$ ) in the absence of pharmacological evidence or strong reasons to justify a preferred assignment or "one-way" interaction. Although both models C1 and C2 were able to characterize the experimental data (Fig. 3A) with acceptable precisions (Table 3), the PD parameters estimated by using model $\mathrm{C} 1\left(k^{\prime}{ }_{1}=1.001 /\right.$ day and $\left.\Psi_{\text {Dox }}=2.78\right)$ were totally different from those with model C2 $\left(k^{\prime}{ }_{1}=0.4511\right.$ day and $\left.\Psi_{\text {Sor }}=1.56\right)$. Since visual inspections of observed data versus model-predicted profiles (Fig. 3A) as well as the precision error values (i.e., \%CV) (Table 3) were comparable between models $\mathrm{C} 1$ and $\mathrm{C} 2$, one model cannot be chosen over the other, as was commonly done in previous studies (Koch

\section{TABLE 2}

PD parameters determined for Dox (intravenous) and Sor (p.o.) monotherapy in xenograft mouse models ( $n=7$ /group)

Values are mean with \%CV. Respective objective function values are included.

\begin{tabular}{lccr}
\hline & Control & Dox $^{\mathrm{a}}$ & \multicolumn{1}{c}{ Sor $^{\mathrm{a}}$} \\
\hline Estimated parameters $^{\mathrm{a}}$ & & & \\
$L_{0}[1 / \mathrm{d}]$ & $0.107(8.40)$ & 0.107 (fixed) & 0.107 (fixed) \\
$L_{1}\left[\mathrm{~cm}^{3} /\right.$ day $]$ & $0.148(0.809)$ & 0.148 (fixed) & 0.148 (fixed) \\
$w_{0}\left[\mathrm{~cm}^{3}\right](\% \mathrm{CV})$ & $0.0412(9.63)$ & $0.0386(10.9)$ & $0.0415(12.9)$ \\
$k_{1}[1 /$ day] $(\% \mathrm{CV})$ & - & $0.130(6.00)$ & $17.0(36.4)$ \\
$k_{2}[\mathrm{l}$ mg per day] $(\% \mathrm{CV})$ & - & $13.8(8.91)$ & $0.0267(35.8)$ \\
Objection function & & & \\
values & 0.0777 & 0.148 & 0.149 \\
MSE & 2.49 & 4.72 & 4.78 \\
SSE & 12.9 & 35.3 & 35.8 \\
AIC & & & \\
\hline
\end{tabular}

$k_{1}$, transient rate constant; $k_{2}$, potency of the drug; $w_{0}$, initial tumor volume prior to administration.

${ }^{a}$ The $L_{0}$ and $L_{1}$ were fixed when estimating PD parameters for Dox and Sor monotherapy. 
TABLE 3

PD parameters determined for Dox plus Sor combination therapy in mice $(n=5)$ by using conventional PK/PD model C and the present new model D (Fig. 1)

Shown are mean values with \%CV. Respective objective function values are included. There are two possibilities (models C1 and C2) when using model C.

\begin{tabular}{|c|c|c|c|}
\hline & Model C1 & Model C2 & Model D \\
\hline \multicolumn{4}{|c|}{ Estimated parameters ${ }^{\mathrm{a}}$} \\
\hline$w_{0}\left[\mathrm{~cm}^{3}\right]$ & $0.0382(15.6)$ & 0.0405 & $0.0422(16.0)$ \\
\hline$k_{1}^{\prime}[1 /$ day $]$ & $1.00(0.0120)$ & $0.451(69.6)$ & $0.832(54.4)$ \\
\hline$\Psi_{\text {Dox }}[-]^{b}$ & $2.78(9.77)$ & - & - \\
\hline$\Psi_{\text {Sor }}[-]^{b}$ & - & $1.56(21.9)$ & - \\
\hline$\alpha[-]$ & - & - & $0.644(11.7)$ \\
\hline$\beta[-]$ & - & - & $1.62(31.8)$ \\
\hline \multicolumn{4}{|c|}{ Objection function values } \\
\hline MSE & 0.129 & 0.120 & 0.130 \\
\hline SSE & 2.84 & 2.64 & 2.72 \\
\hline AIC & 22.8 & 20.9 & 23.8 \\
\hline \multicolumn{4}{|c|}{ Calculated parameter } \\
\hline CI $[-]^{\mathrm{c}}$ & 0.531 & 0.680 & 0.694 \\
\hline
\end{tabular}

$\Psi$, interaction factor; $\alpha$, contribution factor of Dox; $\beta$, contribution factor of Sor; CI, combination index; $k_{1}$, transient rate constant; $w_{0}$, initial tumor volume prior to administration.

${ }^{a} L_{0}\left(0.1071 /\right.$ day) and $L_{1}\left(0.148 \mathrm{~cm}^{3} /\right.$ day) were fixed (Table 2$)$.

${ }^{b}$ Note that distinct $\Psi_{\text {Dox }}$ and $\Psi_{\text {Sor }}$ values were obtained.

${ }^{c} \mathrm{CI}$ values were calculated by using the Chou-Talalay method (Compusyn) for the determination of combination effects.

et al., 2009; Pawaskar et al., 2013; Yuan et al., 2015; Li et al., 2016; Nanavati and Mager, 2017; Chen et al., 2018). Most importantly, the two sets of PD parameters will undoubtedly lead to distinct predictions, as shown in the following studies.

Recognizing that "apparent" contributions from individual drugs to the overall therapeutic outcome may be affected by each other while the intrinsic potency of each drug remains unchanged, we thus developed a new PK-PD model for combination therapy by introducing contribution factors for individual drugs (e.g., $\alpha$ and $\beta$; Fig. 1D). Among a small set of data $(n=7)$, five mice were randomly chosen for model development, whereas two others were left for initial verification. This new PK-PD model D (Fig. 1D) was revealed to nicely characterize the experimental tumor-growth profiles (Fig. 3B). Newly introduced contribution factors were identifiable with current set of data, and the estimated PD parameters (Table 3) showed good precisions. Interestingly, the contribution factors of Dox $(\alpha)$ and $\operatorname{Sor}(\beta)$ values were revealed as 0.644 and 1.62 , respectively, suggesting that Sor had a greater contribution to the overall therapeutic outcome than Dox during combination treatment.

Verification of the New PK-PD Model. The new PK-PD model of combination therapy (Fig. 1D) was evaluated by comparing the simulated tumor progression profile with observed data from two mice (test set; $n=2$ ) randomly left from the set of data. The simulated tumor-growth profiles using the new PK-PD model (Fig. 1D) and estimated PD parameters (Table 3) reasonably characterized the experimental data obtained in the two mice (Supplemental Fig. 2). The $R^{2}$ of linear regression was 0.8565 . and $\mathrm{R}^{2}$ in the Pearson correlation was 0.9632 with a $P$ value of 0.0030 . Similar as the data used for model development (Fig. 3B), the test set of data (Supplemental Fig. 2) showed a greater degree of suppression of tumor growth than Dox and Sor monotherapy (Fig. 2B). The results demonstrate that our new PK-PD model, although the sample size is small, is able to quantitatively describe the antitumor effects of Dox plus Sor combination therapy in xenograft mouse models.

Comparison of Simulations Using the New and Conventional PK-PD Models. Tumor progression profiles in mice treated with various doses of drug combinations were thus simulated by using new model $\mathrm{D}$ and compared with those obtained from conventional models $\mathrm{C} 1$ and C2 (Fig. 4).
As expected, models $\mathrm{C} 1$ and $\mathrm{C} 2$ led to different predictions for the same dose combination of drugs (Fig. 4A), which are simply determined by the distinct interaction factor $\psi$ value estimated for either Dox or Sor. As an example, when Dox dose is doubled and Sor dose is reduced by half (2Dox plus 0.5Sor), model $\mathrm{C} 1$ forecasts a slightly stronger inhibition of tumor growth than Dox plus Sor dose combination (experimental), whereas model C2 predicts a relatively weaker inhibition of disease progression than Dox plus Sor combination (Fig. 4A). Overall, model C1 suggests that tumor growth is sensitive to the changes of both Dox and Sor doses. By contrast, model C2 implies that tumor progression is insensitive to the change of Dox dose, and a greater degree of tumor inhibition may be achieved by the increase of Sor dose.

On the other hand, our new PK-PD model D offered one definitive prediction of disease progression in mice treated with a given dose combination (Fig. 4B). The degree of tumorgrowth inhibition is obviously enhanced with the increase of either Dox or Sor or both drugs simultaneously, although tumor growth seems less sensitive to the change of Dox doses during combination therapy (Supplemental Fig. 3). Interestingly, overall predictions with model $\mathrm{D}$ are in agreement with those by using model C2 (Fig. 4), pointing to a bigger contribution from Sor to overall therapeutic outcome during combination therapy, which is also indicated by a much greater contribution factor of Sor $(\beta=1.62)$ than Dox $(\alpha=$ 0.644 ). In addition, the simulated results suggest that $0.5 \mathrm{Dox}$ plus 2Sor combination would be an optimal dose combination to achieve a greater therapeutic outcome (Fig. 4B).

Evaluation of Pharmacologic Synergism. In doubt about the validity of using interaction factor $\psi$ (Fig. 1C) for the prediction of synergism beyond signifying the degree of change in antitumor effects (Koch et al., 2009), we further employed the Chou-Talalay method or isobologram (Chou, 2006; Tallarida, 2006; Chou, 2010) to calculate the CI values for critical assessment of possible synergism (CI $<1.0$ ), additivity $(\mathrm{CI}=1.0)$, or antagonism $(\mathrm{CI}>1.0)$ (Fig. 1D). The CI value for experimental Dox plus Sor combination therapy is 0.694 (Fig. 4; Table 3), indicating the presence of synergy. The CI values for simulated combination therapy data (Fig. 4) were also calculated for comparison with the utility of $\psi$ 
A Convention Model C: two possibilities Prediction by Model C1
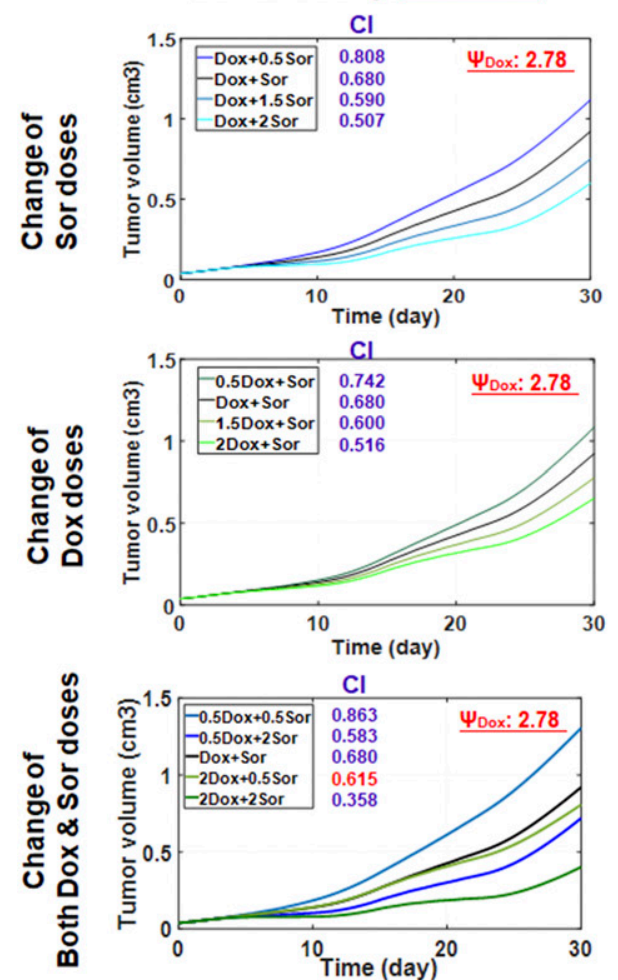
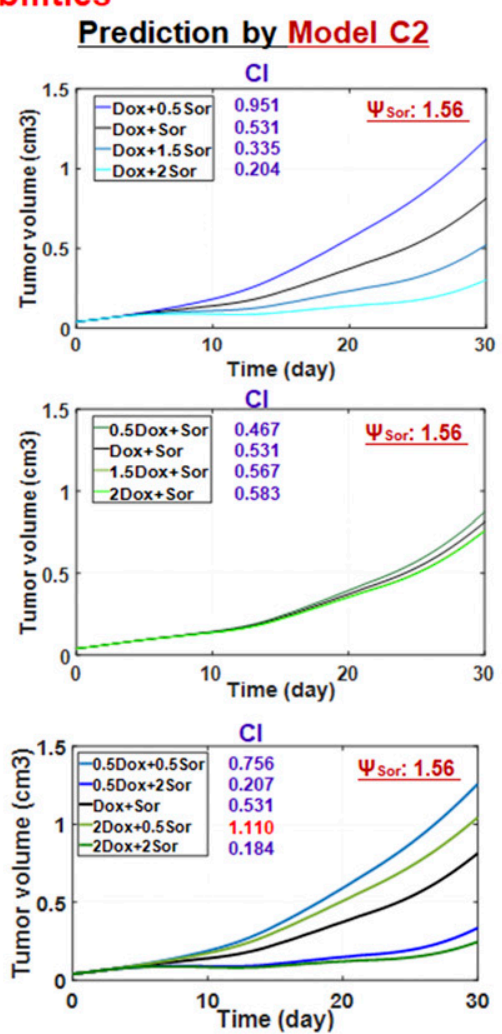

\section{B New Model D: one result} Prediction by Model D
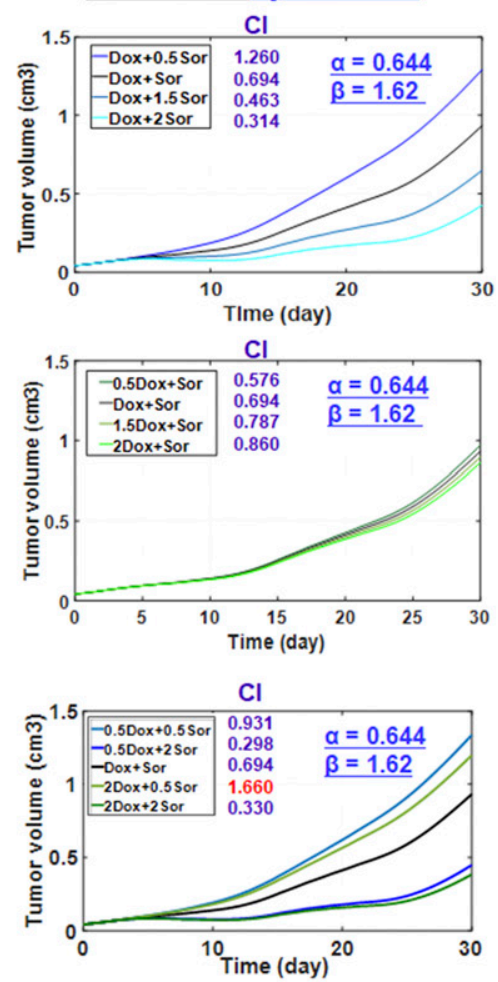

Fig. 4. Comparison of tumor-growth profiles simulated by using conventional PK-PD model C and new model D in response to various doses combinations. CI values were also calculated for individual combinations. (A) Models C1 and C2 provided different predictions of disease progression in mice treated with the same doses of drugs (e.g., 0.5Dox plus 2Sor; 2Dox plus 0.5Sor; etc.), which are largely affected by the distinct interaction factor $\psi$ values estimated for Dox and Sor by models C1 and C2, respectively. The number (e.g., 0.5, 2, 3, or 4) before Dox and Sor means fold change by setting the experimental dose used in this study as 1 (i.e., Dox + Sor). (B) New PK-PD model D offered one definitive result for a given dose combination because the contributions from individual drugs (contribution factors $\alpha$ and $\beta$ ) are taken into consideration. In addition, the calculated CI values vary with the doses of individual drugs used for combination treatment. Among them, certain combinations may produce synergistic effects $(\mathrm{CI}<1)$, whereas others could be antagonistic $(\mathrm{CI}>1)$ or additive $(\mathrm{CI}=1)$, which cannot be defined simply by the same one interaction factor $\psi$ in model C1 or C2. Interestingly, predictions from our model are closer to those from model C2, suggesting a greater contribution from Sor than Dox $(\beta>\alpha)$ to overall therapeutic outcomes during combination treatments.

values. Undeniably, a constant $\psi$ value, either $\psi_{\text {Dox }}$ or $\psi_{\text {Sor }}$, failed to indicate the variable combination effects with the changes of Dox or Sor dose or both (Fig. 4A). By contrast, the CI values were revealed to be variable under different combination treatments, indicating pertinent changes in combination effects. Indeed, when Dox dose is fixed, the CI values decrease with the increase of Sor dose, leading to stronger synergistic effects that cannot be indicated by the constant $\psi$ value (Fig. 4). Moreover, although model C2 offers similar prediction as model $\mathrm{D}$ for the disease progression under 2Dox plus 0.5Sor combination treatment, the constant $\psi_{\text {Sor }}$ value (1.56) ubiquitously suggests the occurrence of synergy, which is in contrast to a slight antagonism defined by using CI value (1.110) (Fig. 4).

To further highlight the importance of utilizing CI value for determination of pharmacologic synergism and irrelevance of using $\psi$ in conventional PK-PD models (Fig. 1), a set of hypothetical data was analyzed, which consisted of five different degrees of assumed tumor-growth profiles (Fig. 5) in mice treated with the same experimental dose combination (i.e., Dox plus Sor). The data were fit to conventional models $\mathrm{C} 1$ and $\mathrm{C} 2$ as well as new model D (Fig. 1), and corresponding PD parameters were estimated (Supplementary Table 1). As expected, the respective contribution factors $\alpha$ and $\beta$ values as well as interaction factors $\psi_{\text {Dox }}$ and $\psi_{\text {sor }}$ differed from each tumor-growth profile in accordance to the distinct extents of tumor inhibition. Likewise, the calculated CI values were different from each other, and a greater degree of tumor suppression (from case 5 to 1 ) was readily indicated by a smaller CI value (from 0.290 to 3.66 ) (Fig. 5). In case 5 , the lowest CI value $(0.290)$ pointed to a strong synergy that seemed to be indicated by both $\psi_{\text {Dox }}$ and $\psi_{\text {Sor }}$ values. However, opposing the antagonism defined by the Chou-Talalay method (CI = 3.66) for case 1 , model $\mathrm{C} 1$ showed a $\psi_{\text {Dox }}$ value (2.16) greater than 1.0, which rather forecasted a synergy (Fig. 5). Most importantly, the $\psi_{\text {Dox }}$ and $\psi_{\text {Sor }}$ values offered different or even opposing assessment of synergism, additivity, and antagonism for the same data (Case 1 or 2; Fig. 5). Together, the results indicate that in addition to two possible predictions, the interaction factors $\left(\psi_{\text {Dox }}\right.$ and $\psi_{\text {Sor }}$ ) in conventional PK-PD models (models C1 and C2; Fig. 1C) are unable to describe variable combination effects for different doses and may provide conflicting assessments, and the CI values should be calculated to critically determine pharmacologic synergism, additivity, and antagonism.

\section{Discussion}

Combination therapy is a common approach to combat against lethal cancers (Webster, 2016), as one drug acting on 


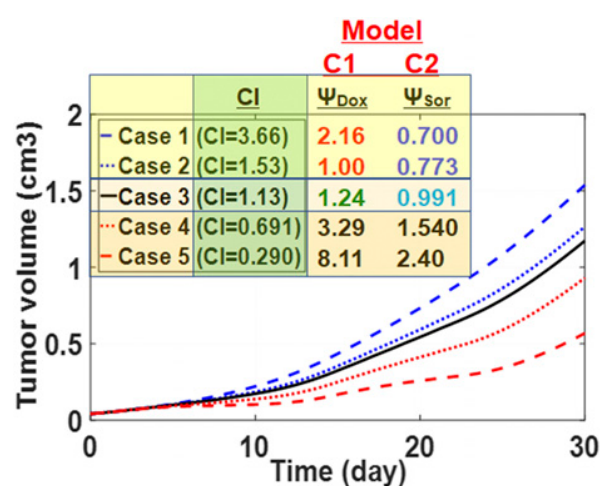

Fig. 5. Variable CI values are obtained for the assessment of different combination effects by using hypothetical tumor-growth data in mice subjected to the same dose combination (Dox + Sor). The interaction factor $\psi$ values determined in models $\mathrm{C} 1$ and $\mathrm{C} 2$ are included for comparison. Note that prediction of synergy using $\psi$ value is inconsistent with the combination index method, and even opposite results may be obtained by using $\psi_{\mathrm{A}}$ vs. $\psi_{\mathrm{B}}$ (or $\psi_{\text {Dox }}$ vs. $\psi_{\text {Sor }}$ ) for the same tumor inhibition data (e.g., case 1).

single target or pathway is usually less effective or subjected to resistance. DNA-intercalating Dox is a potent chemotherapeutic drug with dose-limiting cardiotoxicity (Renu et al., 2018), and Sor is a multikinase inhibitor approved for the treatment of various types of carcinomas (Strumberg, 2005). Dox plus Sor combinations have been evaluated in the clinic and preclinical settings for the treatment of advanced tumors (Abou-Alfa et al., 2010, 2019; Erhardt et al., 2014; Jian et al., 2017). As previous clinical findings that addition of Sor to Dox therapy greatly increased the therapeutic outcomes, whereas addition of Dox to Sor did not show any significant improvement (Abou-Alfa et al., 2010, 2019) were unexplained, our preclinical data and new PK-PD model of combination therapy developed in the present study uncovered a much greater contribution from Sor to the overall efficacy than Dox when coadministered. The contribution terms introduced into this new PK-PD model provide quantitative measurement of apparent contributions from individual drugs to overall outcome of combination treatment. This is distinguished from conventional model involving a single interaction factor that is often assigned to a particular drug (Koch et al., 2009; Pawaskar et al., 2013; Yuan et al., 2015; Li et al., 2016; Nanavati and Mager, 2017; Chen et al., 2018). Our findings demonstrate the utility of this new PK-PD model (model D) in understanding pharmacological interactions and identifying optimal dosages for combination therapy.

Although Dox exhibits a stronger potency than Sor (as manifested by the $k_{2}$ values), Sor monotherapy induces tumor cell death much quicker than Dox (average time to death $N / k_{1}{ }_{1}$ ). The latter can be accounted that the kinetic events intransit compartment model may be the rate-limiting step for tumor-growth inhibition (Simeoni et al., 2004; Pawaskar et al., 2013). As another parameter to describe the overall antitumor effects, time efficacy index (TEI) introduced by Simeoni et al. (2004) can be directly determined from the tumor-growth curve or calculated by using eq. 22 .

$$
T E I=\frac{k_{2} \cdot A U C}{L_{0}}
$$

The TEI values of Dox and Sor treatment groups were 4.67 and 7.20 days at tumor volume of $0.85 \mathrm{~cm}^{3}$, respectively, indicating the degrees of tumor-growth delay or inhibition by the drugs. In addition, suppression of tumor-growth rate is in proportion to $k_{2}$ (i.e., as an index of drug potency) and $C(\mathrm{t}) \cdot x_{1}(\mathrm{t})$ [i.e., accumulative drug exposure or area under of plasma concentration versus time curve (AUC)] (Simeoni et al., 2004). Since " $k_{2} \cdot A U C$ " is equal to "TEI. $L_{0}$ " (eq. 22 ), and $L_{0}$ remains the same, the $k_{2 \mathrm{~A}} \cdot A U C_{\mathrm{A}}$ of Dox should be smaller than $k_{2 \mathrm{~B}} \cdot A U C_{\mathrm{B}}$ of Sor. The latter is in concord to safe exposure to much high levels of Sor, as compared with Dox. These results demonstrate the importance of quantitative measurement of both drug-potency parameters (PD, such as $k_{2}$ ) and drug exposure (PK, such as AUC) as well as other disease and physiologic factors toward a complete understanding and prediction of therapeutic outcomes.

Dox plus Sor treatment at the tested dose combination was much more effective than monotherapy, as indicated by a greater TEI value of 10.5 days at tumor volume of $0.85 \mathrm{~cm}^{3}$. With the development of new PK-PD model (model D), a new combination factor $(\delta)$ may be introduced to quantitatively determine the degree of change in tumor-growth inhibition by combination therapy, which is calculated by using eq. 23 :

$$
\delta=\frac{\boldsymbol{\alpha} \cdot \boldsymbol{k}_{2 A} \cdot \boldsymbol{A U} \boldsymbol{C}_{\boldsymbol{A}}+\boldsymbol{\beta} \cdot \boldsymbol{k}_{2 B} \cdot \boldsymbol{A U} \boldsymbol{C}_{\boldsymbol{B}}}{\boldsymbol{k}_{2 A} \cdot \boldsymbol{A U} \boldsymbol{C}_{\boldsymbol{A}}+\boldsymbol{k}_{2 B} \cdot \boldsymbol{A U} \boldsymbol{C}_{\boldsymbol{B}}}
$$

When the " $k_{2} \cdot A U C$ " is substituted by "TEI. $L_{0}$ " for drugs A and $\mathrm{B}$ (eq. 22) and then the $L_{0}$ is canceled, eq. 24 is derived. Per se, combination factor $\delta$ value may be calculated alternatively with respective $T E I$ values directly obtained from the tumorgrowth curves (Simeoni et al., 2004).

$$
\delta=\frac{\boldsymbol{\alpha} \cdot \boldsymbol{T E I _ { A }}+\boldsymbol{\beta} \cdot \boldsymbol{T E I _ { B }}}{\boldsymbol{T E I _ { A }}+\boldsymbol{T E I _ { B }}}
$$

Combination factor $\delta$ greater than 1 indicates an increase, enhancement, or potentiation of efficacy; less than 1 signifies a decrease, reduction or diminishment of response; and equaling to 1 simply shows a lack of change during combination treatment.

Synergism of combined drugs is based on the concept of equivalent dose and should be critically determined with correct algorisms, whereas simple recognition of " $\mathrm{A}+\mathrm{B}>\mathrm{A}$ " or " $\mathrm{A}+\mathrm{B}>\mathrm{B}$ " does not necessarily indicate a pharmacologic synergy by mixing it up with enhancement or potentiation effects (Chou, 2006). The interaction factor $\psi$ in conventional PK-PD model (Koch et al., 2009) readily signifies the degree of change in efficacy for combined drugs, whereas it has been used to indicate synergism (when $\psi>1$ ) (Koch et al., 2009; Pawaskar et al., 2013; Yuan et al., 2015; Li et al., 2016; Nanavati and Mager, 2017; Chen et al., 2018). Actually, in vitro experimental determination of synergy and interaction mechanism is necessary, which may be used to determine the utility of $\psi$. Nevertheless, although a constant interaction factor $\psi$ enables the simulation of tumor-growth inhibition in response to different combinations, it is unable to characterize variable combination effects for different dosage combinations, as exemplified in current study. In addition, the use of interaction factor may lead to an exaggerated prediction of synergy. Therefore, the present study reiterates the application of valid approaches for the definition of synergism, additivity, and antagonism. The Chou-Talalay method, which is equivalent to classic isobologram approach (Chou, 2006; Tallarida, 2006), is integrated into our new PK-PD model 
(model D) for accurate determination of in vivo pharmacologic synergism.

The new PK-PD model D developed in this study clearly reveals a greater contribution from $\operatorname{Sor}(\beta=1.62)$ to the overall tumor-growth inhibition than Dox $(\alpha=0.644)$ when coadministered, which explains previous clinical findings that coadministration of Sor significantly increases the benefits of Dox treatment, but supplemental Dox does not change Sor effects (Abou-Alfa et al., 2010, 2019). Furthermore, a greater Sor contribution ( $\beta$ values) is in accordance with stronger synergy (smaller CI values when $<1.0$ ). By contrast, there are two possibilities by using conventional PK-PD model in which model C1 emphasizes the influence of Dox $\left(\psi_{\text {Dox }}\right)$ on tumorgrowth inhibition, whereas model $\mathrm{C} 2$ projects a greater impact of Sor $\left(\psi_{\text {Sor }}\right)$ during combination therapy. Indeed, model C2 offers similar predictions as the new model $\mathrm{D}$. That is, both the new model D and conventional model $\mathrm{C} 2$ predict that optimal outcomes may be achieved with the increase of Sor dose, whereas the change of Dox dose has minimal impact on tumorgrowth inhibition. Given the fact that the AUC and maximum concentration of doxorubicin are limiting factors in the optimization of Dox doses (Richly et al., 2009; Levis et al., 2017), reducing Dox dose is preferable to avoid dose-dependent cardiotoxicity. Therefore, the optimal dosage combination (0.5Dox plus 2Sor) identified by the new PK-PD model supports the concept of balancing efficacy and toxicity/safety while maintaining strong synergism $(\mathrm{CI}=0.298)$, which warrants experimental verification.

Although the contribution factors introduced in present study are identifiable with good precision, and this new PKPD model of combination therapy was quantified for the proof of concept, the sample size was relatively small (PK data, $n=$ 6; PD data, $n=7$ ), among which the PD data of five individual mice were randomly chosen for model development, and two others were left out and used for model verification. It is necessary to challenge this model with much larger sets of data and perform more extensive model validation and sensitivity analyses in future studies. Given the fact that interactions of coadministered drugs may occur at $\mathrm{PK}$ and/or PD levels, this new PK-PD model might signify overall PK and PD interactions. Rather, the PK data of combined drugs may be collected to recapitulate possible PK interactions, which can be incorporated into the final PK-PD model to define the specific influence of PK interactions on PD outcomes. Actually, PK interactions between Dox and Sor coadministered in patients with hepatocellular carcinoma have been revealed to be negligible (Richly et al., 2009), whereas biliary excretion and cytochrome P450 and uridine diphosphate glucuronosyl transferase-mediated metabolism are recognized as major elimination routes for both Dox and Sor (Choi et al., 2013; Edginton et al., 2016). In addition, the PK data in current study were collected from "healthy" male mice, whereas PD data were obtained from female mice bearing xenograft tumors (Jian et al., 2017). Therefore, the present study might have missed possible influence of xenograft tumors, similar to other diseases or disease statuses (Li et al., 2019), as well as sex (Franconi and Campesi, 2014) on the PK properties of Dox or Sor (e.g., volume of the central compartment), whereas the differences in PK among mouse strains seem minimal (Barr et al., 2020). Indeed, it has been reported that doxorubicin clearance was relatively higher in men $(n=6)$ than in women $(n=21)\left(59 \mathrm{vs} .27 \mathrm{l} \cdot \mathrm{h}^{-1} \cdot \mathrm{m}^{-2}\right)$ (Dobbs et al., 1995), and abnormal liver functions tended to correlate with lower levels of doxorubicin clearance (Twelves et al., 1998). Baseline body weight was identified as a statistically significant covariate for variable sorafenib distributional volume among patients with solid tumors (Jain et al., 2011), whereas no association was found between organ function and systemic sorafenib exposure, including patients with severe liver and kidney impairment (Miller et al., 2009). Rather, sorafenib systemic exposure was revealed to decrease over time in patients with hepatocellular carcinoma (Arrondeau et al., 2012). Actually, differences in PK properties between distinct populations, if any, are generally associated with the variations in albumin levels, liver, or kidney functions (Cheeti et al., 2013; Lacy et al., 2018; Gupta et al., 2020), which may be taken into consideration when developing PK models for particular populations. Ultimately, physiologically based PK-modeling approaches may be used toward translating the PK-PD relationship across species or populations, including the prediction of combined drug effects in patients with cancer (Cheeti et al., 2013; Ande et al., 2018; Garcia-Cremades et al., 2019).

In summary, a new PK-PD model for combination treatment was established in this study by considering apparent contributions from individual drugs coadministered. This PKPD model quantitatively disclosed a much greater contribution from Sor to overall tumor inhibition than Dox during combination therapy, offering explanation for the inexplicable clinical findings (Abou-Alfa et al., 2010, 2019). Furthermore, the Chou-Talalay method was integrated into this predictive PK-PD model to accurately determine in vivo synergism. In addition, optimal dosage combinations could be identified to improve therapeutic outcomes consistent with the strongest synergy. This new PK-PD model and strategy should have broad applications to pharmacological and translational research.

\section{Authorship Contributions}

Participated in research design: Choi, Zhang, Liu, Tu, A.-X. Yu, A.-M. Yu.

Conducted experiments: Choi, Zhang, Liu, Tu.

Contributed new reagents or analytic tools: Choi, A.-M. Yu.

Performed data analysis: Choi, Zhang, Liu, Tu, A.-X. Yu, A.-M. Yu.

Wrote or contributed to the writing of the manuscript: Choi, Zhang, Liu, Tu, A.-X. Yu, A.-M. Yu.

\section{References}

Abou-Alfa GK, Johnson P, Knox JJ, Capanu M, Davidenko I, Lacava J, Leung T, Gansukh B, and Saltz LB (2010) Doxorubicin plus sorafenib vs doxorubicin alone in patients with advanced hepatocellular carcinoma: a randomized trial. JAMA 304: 2154-2160.

Abou-Alfa GK, Shi Q, Knox JJ, Kaubisch A, Niedzwiecki D, Posey J, Tan BR Jr, Kavan P, Goel R, Lammers PE, et al. (2019) Assessment of treatment with sorafenib plus doxorubicin vs sorafenib alone in patients with advanced hepatocellular carcinoma: phase 3 CALGB 80802 randomized clinical trial. JAMA Oncol 5: 1582-1588.

Altrock PM, Liu LL, and Michor F (2015) The mathematics of cancer: integrating quantitative models. Nat Rev Cancer 15:730-745.

Ande A, Chaar M, and Ait-Oudhia S (2018) Multiscale systems pharmacological analysis of everolimus action in hepatocellular carcinoma. J Pharmacokinet Pharmacodyn 45:607-620.

Arrondeau J, Mir O, Boudou-Rouquette P, Coriat R, Ropert S, Dumas G, Rodrigues MJ, Rousseau B, Blanchet B, and Goldwasser F (2012) Sorafenib exposure decreases over time in patients with hepatocellular carcinoma. Invest New Drugs 30:2046-2049.

Ayyar VS and Jusko WJ (2020) Transitioning from basic toward systems pharmacodynamic models: lessons from corticosteroids. Pharmacol Rev 72:414-438.

Barr JT, Tran TB, Rock BM, Wahlstrom JL, and Dahal UP (2020) Strain-dependent variability of early discovery small molecule pharmacokinetics in mice: does strain matter? Drug Metab Dispos 48:613-621.

Cheeti S, Budha NR, Rajan S, Dresser MJ, and Jin JY (2013) A physiologically based pharmacokinetic (PBPK) approach to evaluate pharmacokinetics in patients with cancer. Biopharm Drug Dispos 34:141-154. 
Chen W, Chen R, Li J, Fu Y, Yang L, Su H, Yao Y, Li L, Zhou T, and Lu W (2018) Pharmacokinetic/pharmacodynamic modeling of schedule-dependent interaction between docetaxel and cabozantinib in human prostate cancer xenograft models. $J$ Pharmacol Exp Ther 364:13-25.

Cho YK, Irby DJ, Li J, Sborov DW, Mould DR, Badawi M, Dauki A, Lamprecht M, Rosko AE, Fernandez S, et al. (2018) Pharmacokinetic-pharmacodynamic model of neutropenia in patients with myeloma receiving high-dose melphalan for autologous stem cell transplant. CPT Pharmacometrics Syst Pharmacol 7:748-758.

Choi YH, Lee YK, and Lee MG (2013) Effects of $17 \alpha$-ethynylestradiol-induced cholestasis on the pharmacokinetics of doxorubicin in rats: reduced biliary excretion and hepatic metabolism of doxorubicin. Xenobiotica 43:901-907.

Chou TC (2006) Theoretical basis, experimental design, and computerized simulation of synergism and antagonism in drug combination studies. Pharmacol Rev 58: 621-681.

Chou TC (2010) Drug combination studies and their synergy quantification using the Chou-Talalay method. Cancer Res 70:440-446.

Claret L, Girard P, Hoff PM, Van Cutsem E, Zuideveld KP, Jorga K, Fagerberg J, and Bruno R (2009) Model-based prediction of phase III overall survival in colorectal cancer on the basis of phase II tumor dynamics. J Clin Oncol 27: 4103-4108.

Diekstra MH, Fritsch A, Kanefendt F, Swen JJ, Moes D, Sörgel F, Kinzig M, Stelzer C, Schindele D, Gauler T, et al. (2017) Population modeling integrating pharmacokinetics, pharmacodynamics, pharmacogenetics, and clinical outcome in patients with sunitinib-treated cancer. CPT Pharmacometrics Syst Pharmacol 6:604-613.

Dobbs NA, Twelves CJ, Gillies H, James CA, Harper PG, and Rubens RD (1995) Gender affects doxorubicin pharmacokinetics in patients with normal liver biochemistry. Cancer Chemother Pharmacol 36:473-476.

Edginton AN, Zimmerman EI, Vasilyeva A, Baker SD, and Panetta JC (2016) Sorafenib metabolism, transport, and enterohepatic recycling: physiologically based modeling and simulation in mice. Cancer Chemother Pharmacol 77:1039-1052.

Erhardt A, Kolligs F, Dollinger M, Schott E, Wege H, Bitzer M, Gog C, Lammert F, Schuchmann M, Walter C, et al. (2014) TACE plus sorafenib for the treatment of hepatocellular carcinoma: results of the multicenter, phase II SOCRATES trial. Cancer Chemother Pharmacol 74:947-954.

Franconi F and Campesi I (2014) Pharmacogenomics, pharmacokinetics and pharmacodynamics: interaction with biological differences between men and women. $\mathrm{Br}$ $J$ Pharmacol 171:580-594

Fu J, Zhang N, Chou JH, Dong HJ, Lin SF, Ulrich-Merzenich GS, and Chou TC (2016) Drug combination in vivo using combination index method: taxotere and T607 against carcinoma HCT-116 xenograft tumor in nude mice. Synergy 3:15-30.

Garcia-Cremades M, Pitou C, Iversen PW, and Troconiz IF (2019) Translational framework predicting tumour response in gemcitabine-treated patients with advanced pancreatic and ovarian cancer from xenograft studies. AAPS J 21:23.

Gupta N, Wang X, Offman E, Prohn M, Narasimhan N, Kerstein D, Hanley MJ, and Venkatakrishnan K (2020) Population pharmacokinetics of brigatinib in healthy volunteers and patients with cancer. Clin Pharmacokinet 60:235-247.

Iida H, Fujikawa R, Kozaki R, Harada R, Hosokawa Y, Ogawara KI, and Ohno T (2020) Pharmacokinetic-pharmacodynamic-efficacy modeling of ONO-7579, a novel pan-tropomyosin receptor kinase inhibitor, in a murine xenograft tumor model. $J$ Pharmacol Exp Ther 373:361-369.

Jain L, Woo S, Gardner ER, Dahut WL, Kohn EC, Kummar S, Mould DR, Giaccone G, Yarchoan R, Venitz J, et al. (2011) Population pharmacokinetic analysis of sorafenib in patients with solid tumours. Br J Clin Pharmacol 72:294-305.

Jian C, Tu MJ, Ho PY, Duan Z, Zhang Q, Qiu JX, DeVere White RW, Wun T, Lara PN, Lam KS, et al. (2017) Co-targeting of DNA, RNA, and protein molecules provides optimal outcomes for treating osteosarcoma and pulmonary metastasis in spontaneous and experimental metastasis mouse models. Oncotarget 8 : 30742-30755.

Jilek JL, Tian Y, and Yu AM (2017) Effects of MicroRNA-34a on the pharmacokinetics of cytochrome P450 probe drugs in mice. Drug Metab Dispos 45:512-522.

Jilek JL, Tu MJ, Zhang C, and Yu AM (2020) Pharmacokinetic and pharmacodynamic factors contribute to synergism between let-7c-5p and 5-fluorouracil in inhibiting hepatocellular carcinoma cell viability. Drug Metab Dispos 48 $1257-1263$.

Koch G, Walz A, Lahu G, and Schropp J (2009) Modeling of tumor growth and anticancer effects of combination therapy. J Pharmacokinet Pharmacodyn 36:179-197.

Lacy S, Yang B, Nielsen J, Miles D, Nguyen L, and Hutmacher M (2018) A population pharmacokinetic model of cabozantinib in healthy volunteers and patients with various cancer types. Cancer Chemother Pharmacol 81:1071-1082.

Laird AK (1964) Dynamics of tumor growth. Br J Cancer 13:490-502.

Levis BE, Binkley PF, and Shapiro CL (2017) Cardiotoxic effects of anthracyclinebased therapy: what is the evidence and what are the potential harms? Lancet Oncol 18:e445-e456.

Li J, Jameson MB, Baguley BC, Pili R, and Baker SD (2008) Population pharmacokinetic-pharmacodynamic model of the vascular-disrupting agent 5,6dimethylxanthenone-4-acetic acid in cancer patients. Clin Cancer Res 14: $2102-2110$

Li JY, Ren YP, Yuan Y, Ji SM, Zhou SP, Wang LJ, Mou ZZ, Li L, Lu W, and Zhou TY (2016) Preclinical PK/PD model for combined administration of erlotinib and sunitinib in the treatment of A549 human NSCLC xenograft mice. Acta Pharmacol Sin 37:930-940.
Li Y, Meng Q, Yang M, Liu D, Hou X, Tang L, Wang X, Lyu Y, Chen X, Liu K, et al. (2019) Current trends in drug metabolism and pharmacokinetics. Acta Pharm Sin $B$ 9:1113-1144.

Mager DE, Wyska E, and Jusko WJ (2003) Diversity of mechanism-based pharmacodynamic models. Drug Metab Dispos 31:510-518.

Miller AA, Murry DJ, Owzar K, Hollis DR, Kennedy EB, Abou-Alfa G, Desai A Hwang J, Villalona-Calero MA, Dees EC, et al. (2009) Phase I and pharmacokinetic study of sorafenib in patients with hepatic or renal dysfunction: CALGB 60301. $J$ Clin Oncol 27:1800-1805.

Mould DR, Walz AC, Lave T, Gibbs JP, and Frame B (2015) Developing exposure/ response models for anticancer drug treatment: special considerations. CPT Pharmacometrics Syst Pharmacol 4:e00016.

Nanavati C and Mager DE (2017) Sequential exposure of bortezomib and vorinostat is synergistic in multiple myeloma cells. Pharm Res 34:668-679.

National Research Council (US) Committee for the Update of the Guide for the Care and Use of Laboratory Animals (2011) Guide for the Care and Use of Laboratory Animals, National Academies Press (US), Washington (DC).

Pawaskar DK, Straubinger RM, Fetterly GJ, Hylander BH, Repasky EA, Ma WW, and Jusko WJ (2013) Synergistic interactions between sorafenib and everolimus in pancreatic cancer xenografts in mice. Cancer Chemother Pharmacol 71:1231-1240.

Renu K, v G A, P B TP, and Arunachalam S (2018) Molecular mechanism of doxorubicin-induced cardiomyopathy - an update. Eur J Pharmacol 818:241-253. Richly H, Schultheis B, Adamietz IA, Kupsch P, Grubert M, Hilger RA, Ludwig M, Brendel E, Christensen O, and Strumberg D (2009) Combination of sorafenib and doxorubicin in patients with advanced hepatocellular carcinoma: results from a phase I extension trial. Eur J Cancer 45:579-587.

Rocchetti M, Del Bene F, Germani M, Fiorentini F, Poggesi I, Pesenti E, Magni P, and De Nicolao G (2009) Testing additivity of anticancer agents in pre-clinical studies: a PK/PD modelling approach. Eur J Cancer 45:3336-3346.

Schindler E, Amantea MA, Karlsson MO, and Friberg LE (2016) PK-PD modeling of individual lesion FDG-PET response to predict overall survival in patients with sunitinib-treated gastrointestinal stromal tumor. CPT Pharmacometrics Syst Pharmacol 5:173-181.

Simeoni M, Magni P, Cammia C, De Nicolao G, Croci V, Pesenti E, Germani M Poggesi I, and Rocchetti M (2004) Predictive pharmacokinetic-pharmacodynamic modeling of tumor growth kinetics in xenograft models after administration of anticancer agents. Cancer Res 64:1094-1101.

Strumberg D (2005) Preclinical and clinical development of the oral multikinase inhibitor sorafenib in cancer treatment. Drugs Today (Barc) 41:773-784.

Tallarida RJ (2006) An overview of drug combination analysis with isobolograms. $J$ Pharmacol Exp Ther 319:1-7.

Tanaka C, O'Reilly T, Kovarik JM, Shand N, Hazell K, Judson I, Raymond E, Zumstein-Mecker S, Stephan C, Boulay A, et al. (2008) Identifying optimal biologic doses of everolimus (RAD001) in patients with cancer based on the modeling of preclinical and clinical pharmacokinetic and pharmacodynamic data. J Clin Oncol 26:1596-1602.

Terranova N, Germani M, Del Bene F, and Magni P (2013) A predictive pharmacokinetic-pharmacodynamic model of tumor growth kinetics in xenograft mice after administration of anticancer agents given in combination. Cancer Chemother Pharmacol 72:471-482.

Twelves CJ, Dobbs NA, Gillies HC, James CA, Rubens RD, and Harper PG (1998) Doxorubicin pharmacokinetics: the effect of abnormal liver biochemistry tests. Cancer Chemother Pharmacol 42:229-234.

Vaghi C, Rodallec A, Fanciullino R, Ciccolini J, Mochel JP, Mastri M, Poignard C, Ebos JML, and Benzekry S (2020) Population modeling of tumor growth curves and the reduced Gompertz model improve prediction of the age of experimental tumors. PLOS Comput Biol 16:e1007178.

Wada R, Erickson HK, Lewis Phillips GD, Provenzano CA, Leipold DD, Mai E, Johnson H, and Tibbitts J (2014) Mechanistic pharmacokinetic/pharmacodynamic modeling of in vivo tumor uptake, catabolism, and tumor response of trastuzumab maytansinoid conjugates. Cancer Chemother Pharmacol 74:969-980.

Wang J, Zou JX, Xue X, Cai D, Zhang Y, Duan Z, Xiang Q, Yang JC, Louie MC, Borowsky $\mathrm{AD}$, et al. (2016) ROR- $\gamma$ drives androgen receptor expression and represents a therapeutic target in castration-resistant prostate cancer. Nat Med 22: $488-496$.

Webster RM (2016) Combination therapies in oncology. Nat Rev Drug Discov 15: 81-82.

Yi W, Tu MJ, Liu Z, Zhang C, Batra N, Yu AX, and Yu AM (2020) Bioengineered miR328-3p modulates GLUT1-mediated glucose uptake and metabolism to exert synergistic antiproliferative effects with chemotherapeutics. Acta Pharm Sin B 10: 159-170.

Yuan Y, Zhou X, Ren Y, Zhou S, Wang L, Ji S, Hua M, Li L, Lu W, and Zhou T (2015) Semi-mechanism-based pharmacokinetic/pharmacodynamic model for the combination use of dexamethasone and gemcitabine in breast cancer. J Pharm Sci 104: 4399-4408.

Address correspondence to: $\mathrm{Dr}$. Ai-Ming $\mathrm{Yu}$, Department of Biochemistry and Molecular Medicine, UC Davis School of Medicine, Sacramento, CA 95817. E-mail: aimyu@ucdavis.edu 